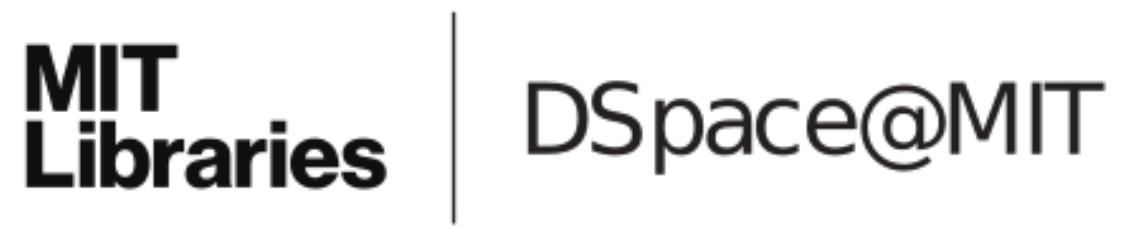

\author{
MIT Open Access Articles
}

Prehensile Pushing: In-hand Manipulation with Push-Primitives

The MIT Faculty has made this article openly available. Please share how this access benefits you. Your story matters.

Citation: Chavan-Dafle, Nikhil, and Alberto Rodriguez. "Prehensile Pushing: In-hand Manipulation with Push-Primitives." 2015 IEEE/RSJ International Conference on Intelligent Robots and Systems (September 2015).

As Published: https://ras.papercept.net/conferences/scripts/abstract.pl? ConfID $=103 \&$ Number $=1617$

Publisher: Institute of Electrical and Electronics Engineers (IEEE)

Persistent URL: http://hdl.handle.net/1721.1/98114

Version: Author's final manuscript: final author's manuscript post peer review, without publisher's formatting or copy editing

Terms of use: Creative Commons Attribution-Noncommercial-Share Alike 


\section{Prehensile Pushing: In-hand Manipulation with Push-Primitives}



\begin{abstract}
This paper explores the manipulation of a grasped object by pushing it against its environment. Relying on precise arm motions and detailed models of frictional contact, prehensile pushing enables dexterous manipulation with simple manipulators, such as those currently available in industrial settings, and those likely affordable by service and field robots.

This paper is concerned with the mechanics of the forceful interaction between a gripper, a grasped object, and its environment. In particular, we describe the quasi-dynamic motion of an object held by a set of point, line, or planar rigid frictional contacts and forced by an external pusher (the environment). Our model predicts the force required by the external pusher to "break" the equilibrium of the grasp and estimates the instantaneous motion of the object in the grasp. It also captures interesting behaviors such as the constraining effect of line or planar contacts and the guiding effect of the pusher's motion on the objects's motion.
\end{abstract}

We evaluate the algorithm with three primitive prehensile pushing actions-straight sliding, pivoting, and rolling-with the potential to combine into a broader in-hand manipulation capability.

\section{INTRODUCTION}

The ability to adjust a grasp on an object, seemingly simple for humans but still out of reach for robots, is both essential and commonplace. For example we adjust the grip on a hammer before hammering a nail, and change the grip on the nail in expectation of the stroke of the hammer. The human hand surely plays an important role; however, it is through the effective use of hands, arms, and the environment around us that we become proficient.

This paper exploits contacts with the environment, rather than gripper dexterity, to manipulate a grasped object. We refer by prehensile pushing to the act of pushing a grasped object against the environment to manipulate it. It relies on precise arm motions to provide the necessary level of dexterity and fine manipulation, circumventing the need for complex finger motions. From the hand's point of view the

This work was supported by NSF award [NSF-IIS-1427050] through the National Robotics Initiative and by the Karl Chang Innovation Fund Award. environment is a precise and highly dexterous virtual finger whose motion is a reflection of the motion of the arm, forcing the object to move through a series of stable grasps. In [1] we refer to this kind of dexterity as extrinsic dexterity .

The goal of our work is to demonstrate that prehensile pushing is an efficient and robust solution to bring dexterity to robotic manipulation, of special relevance to applications that demand solutions without complex or expensive hardware, such as automation, service, or field robotics.

A canonical prehensile push is composed of four phases: i) The object begins in a stable grasp withstanding gravity; ii) the grasp is augmented with an external contact, which adds stability and enables the relaxation of the gripping force; iii) a controlled push reconfigures the object; and iv) the external contact is released, possibly requiring an increase in the gripping force. This paper formulates an algorithm to describe the pushing phase. The key contributions are:

- A formulation that captures the development of forces and accelerations at all contacts-internal and external-on a grasped object, and characterizes the instant when the stability of the grasp breaks leading to a grasp adjustment;

- a computationally tractable approach to model point, line, and planar patch contacts, that captures their kinematic constraining effect, linear and rotation friction, and the guiding effect of the pusher's motion;

- and an experimental validation of the predictions of our model on three primitive actions: straight sliding, pivoting, and rolling.

We formulate the interaction between gripper, object and external pusher as a complementarity problem (Trinkle et al. [2]) subject to kinematic and kinetic constraints on the motion of the bodies in contact. We adopt a conventional linear approximation of friction cones (Goldman and Tucker [3]) and solve for the complementarity constraints as a quadratic optimization problem in the space of local forces 

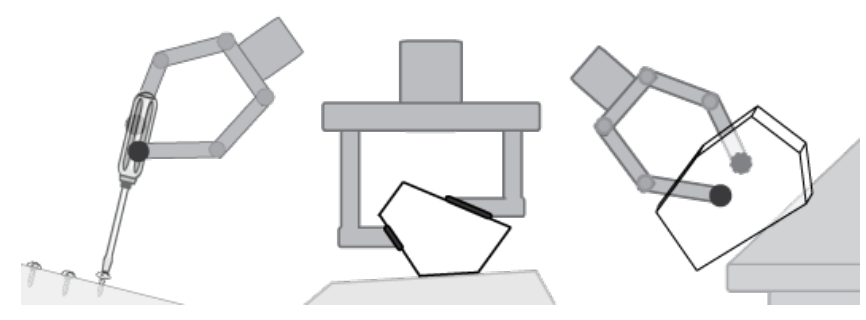

Fig. 1. Examples of prehensile pushes: driving a screw, pushing against the ground, and pivoting about an edge.

and relative accelerations at all contacts. The solution provides the pushing force required to break the grasp and the consequent instantaneous twist of the object. The formulation is general over objects with known inertia and geometry, over grippers with known kinematics, over variations on contact configurations such as number or type of contacts, and over motions of the pusher. Fig. 1 illustrates some examples.

In this paper, we apply the formulation to the study of three primitive pushing actions — straight sliding, pivoting and rolling-with a two-finger parallel jaw gripper and a variety of pushers. For each action, we investigate the sensitivity of the force required to move the object and the resultant object twist, to variations in the location of contact, the type of contact geometry, and the motion of the pusher. We conclude by arguing that a suit of stable prehensile pushing primitives can serve as building blocks towards a broad and versatile approach to in-hand manipulation.

\section{RELATED WORK}

The robotics community, very aware of the key role of dexterity in autonomous manipulation, has chased it since the 1980s. The so called "dexterous hands" approach to robotic manipulation started with Salisbury [4, 5], and has been thoroughly analyzed since then $[6,7,8]$. It focuses on the manipulation of an object held and actuated through finely controlled fingertip contacts. It leads to elegant but complex solutions $[9,10,11]$ and consequently faces limitations such as hardware and control complexity and limited range of motions.

Humans, in addition, enhance their dexterity with a wide and wild set of tricks. Gravity, high accelerations, or the environment, all have the potential to assist manipulation. In previous work [1] we demonstrated a series of hand coded in-hand manipulation actions that exploit resources external to the hand. We refer to the approach as extrinsic dexterity and classify them into three main categories: quasi-static or quasi-dynamic actions powered by external contacts, passivedynamic actions that exploit gravity, and active-dynamic actions powered by fast motions of the arm. The work in this paper belongs to the first category, where we manipulate a grasped object by pushing it against a fixed environment.

There is a significant amount of work on modeling the relationship between forces and motions for sliding and rolling contact interactions [5, 12, 13, 14, 15], all providing in some way or another contact models, characterizations of the geometric and frictional constraints induced by contact, and their effects on grasp stability and object motion.

In particular, the idea of allowing contact slip to enhance in-hand manipulation has been explored in the context of prehensile manipulation $[11,16,17,18,19]$. Specially related to our work, Brock [11] studies reorienting a grasped object using contacts from the environment. He relies on an "idealized" external wrench derived from figurative external point contacts which are assumed to always stick to the object. In our work, we incorporate the particularities of that external wrench being generated by a real external pusher, and solve for local contact forces and relative accelerations at the external contacts. Moreover, we consider complex contact interactions beyond simple point contact models.

Hong and Cutkosky [20] study the stability of a fixtured part against a tool force, and provide estimates for the fixture gripping force required to prevent slippage of the part. A quasi-static analysis suffices for their analysis, given that their goal is to prevent slippage by maintaining force balance. Our formulation is quasi-dynamic to capture the effect of the motion of the pusher and the force imbalance it causes leading to deliberate slippage at one or more contacts. They assume a known contact force at the tool application point as Brock does which does not capture the indeterminacy due to friction between object and tool.

Finally, prehensile pushing is also closely related to its non-prehensile counterpart, where a planar object is pushed on a flat frictional surface. The indeterminacy of the pressure distribution across the surface contact between the object and the ground limits the set of motions that are controllable and predictable by the pusher [14, 21]. Lynch and Mason [22, 23] successfully determine bounds on the set of stable pushes for a given flat object. Prehensile pushing can be seen as analogous to non-prehensile pushing, where the pressure points are known and located at the finger contacts, and where we resolve the corresponding pressure distribution through grasp optimization.

\section{The Prehensile Pushing Problem}

The main goal of this work is to predict the motion of a grasped object when pushed against a fixed environment through a controlled motion of the manipulator.

For simplicity of exposition, we formulate the equivalent problem of an object grasped by a fixed hand and pushed by a moving environment. The virtual motion of the environment is a reflection of the motion of the arm, as seen from a reference frame on the hand. We make the assumption that the arm moving the object has full cartesian dexterity-quite reasonable for a typical robotic manipulator with 6 or more degrees of freedom-which in our equivalent formulation, gives the external pusher/environment the freedom to push in any direction and orientation.

Figures 2 and 3 illustrate a real and a schematic version of a simple planar prehensile push. In the following sections we detail further the prehensile pushing problem and outline our approach to solve it. 


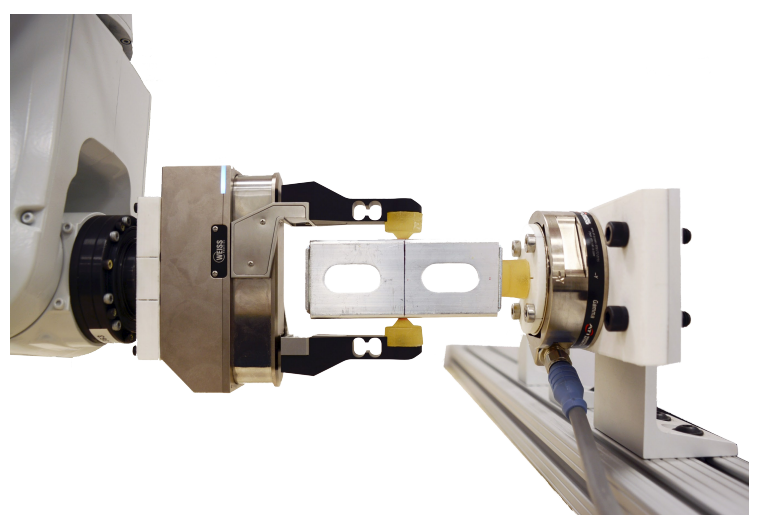

Fig. 2. Example of a prehensile push: a two-finger gripper pushing an object against a line feature in the environment.

\section{A. Problem Formulation}

We assume the following information:

- the shape and mass of the object;

- the location, geometries, and frictional properties of all contacts between the object and the gripper;

- the kinematics of the gripper, in the form of the jacobian from actuators to contact velocities;

- the magnitude of the gripping forces; and

- the location, geometry, frictional properties, and motion of the external pusher.

In the simplified planar case illustrated in Fig. 3 a paralleljaw gripper with simple open-close kinematics is holding a prismatic object with two point contacts, each capable of transmitting normal forces $\left(\vec{f}_{1_{n}}\right.$ and $\left.\vec{f}_{2_{n}}\right)$ and frictional forces tangent to the object surface $\left(\vec{f}_{1_{t}}\right.$ and $\left.\vec{f}_{2_{t}}\right)$. The external pusher moving to the left, makes a line frictional contact with the object, and is capable of transmitting normal force $\vec{f}_{\mathrm{ext}_{n}}$, frictional force $\vec{f}_{\text {ext }_{t}}$, and balancing moments $\vec{m}_{\text {ext }}$ along axes off from the line.

Note that in the three dimensional case, each contact will exert additional frictional forces and moments based on the contact model. Also note that contacts with nontrivial geometry, such as a line or a planar contact (Fig. 4), change grasp analysis at least in two key ways: kinematically, the contact geometry constrains the set of relative motions between two bodies more than a point contact would do; and kinetically, the normal and frictional contact forces can be asymmetrically distributed across the patch, leading to anisotropy in the "effective" frictional force. The method in this paper captures both the effects.

For the particular case of Fig. 3, we are interested in finding the minimum normal force at the pusher $\vec{f}_{\operatorname{ext}_{n}}$ that will move the object in the grasp, and in characterizing the instantaneous direction of motion of the object.

\section{B. Approach}

The following sections formulate the analysis of forces and motions at contacts as a complementarity problem in the spirit of Trinkle et al. [2], subject to a series of extra

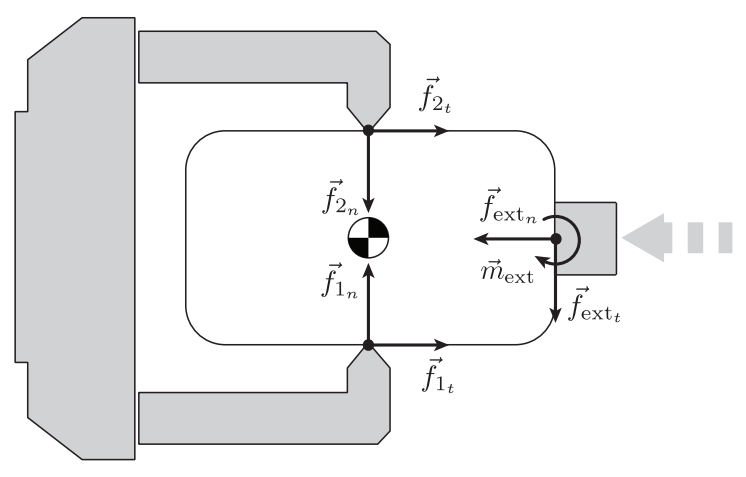

Fig. 3. Planar schematic of the prehensile push in Fig. 2. In this formulation the environment moves as a reflection of the motion of the arm.

linear constraints. More precisely, the problem is formulated in the space of:

- $\vec{f}_{\boldsymbol{i}}, \vec{f}_{\text {ext }}$ : forces at all contact points between the gripper, the object, and the external pusher.

- $\overrightarrow{\boldsymbol{a}}_{\boldsymbol{i}}, \overrightarrow{\boldsymbol{a}}_{\text {ext }}$ : relative accelerations at each contact.

- $\overrightarrow{\boldsymbol{a}}_{\text {obj }}$ : acceleration of the object.

And is subject to the following constraints:

- Newtonian mechanics: The acceleration of the object results from the total wrench applied on the object by the internal grasp forces, the forces at the external pusher, and gravity.

- Rigid body constraints: Accelerations at contacts should be in accordance with that of the object.

- Unilateral contact constraints: Contacts can only push, and only if the contact is maintained.

- Frictional force constraints: The normal force, tangential frictional force, and tangential acceleration at each contact should follow Coulomb's friction law and the principle of maximum energy dissipation.

- Constraints due to complex contacts: We model contacts with non trivial geometry as a discrete set of rigidly attached frictional points. The individual interactions of each constituent point with the object must respect the rigidity of the patch.

- Constraints due to the motion of the pusher: When the grasp breaks, the object must follow the pusher motion. In particular, their relative normal accelerations at contact must comply.

We solve the resulting mixed complementarity problem by minimizing the quadratic complementarity constraints to zero subject to extra kinematic and frictional linear constraints. Section IV and Section V describe in more detail each individual constraint, and Section VI gives the complete formulation.

\section{Contact Modeling}

This section starts with a short review on conventional point contact models and the standard linearization of Coulomb friction cone for point contacts, and then describes our approach to model contacts with complex geometries. 


\section{A. Point Contact Models}

It is challenging to accurately model frictional interaction. Contact models, however, are compact and computationally convenient, and to some degree indicative. At the most basic level, contact models encode the directions along which a contact can transmit forces and torques [24]. We list here the three most common:

- Frictionless point contact: It transmits force only along the normal to the surface.

- Frictional hard point contact: It transmits forces along three directions-one normal and two tangential—but no torques. It approximates interaction with a small contact area.

- Frictional soft point contact: It transmits forces along three directions-one normal and two tangential-and torque about the contact normal. It approximates interaction with a larger contact area.

In the presence of friction, the tangential and normal transmitted forces are related by Coulomb's law, and the coefficient of friction $\mu$.

Consider a contact between a finger and an object, and a frame at contact composed by unit vectors $\hat{\boldsymbol{n}}$ normal to the surface, and $\hat{\boldsymbol{t}}$ and $\hat{\boldsymbol{o}}$ both orthogonal and spanning the tangent plane. Let $\lambda_{n}, \lambda_{t}$, and $\lambda_{o}$ be the magnitudes of the normal force, and frictional forces along $\hat{\boldsymbol{t}}$ and $\hat{o}$ respectively. We express the total contact force in the local coordinates $\langle\hat{\boldsymbol{n}}, \hat{\boldsymbol{t}}, \hat{\boldsymbol{o}}\rangle$ as $\boldsymbol{\Lambda}=\left[\lambda_{n}, \lambda_{t}, \lambda_{o}\right]^{\top}$. Coulomb's friction law is satisfied if the total contact force is inside the following set:

$$
F C=\left\{\lambda_{n} \hat{\boldsymbol{n}}+\lambda_{t} \hat{\boldsymbol{t}}+\lambda_{o} \hat{\boldsymbol{o}} \mid \lambda_{n} \geq 0, \lambda_{t}^{2}+\lambda_{o}^{2} \leq \mu \lambda_{n}^{2}\right\}
$$

known as friction cone, where the last inequality becomes a strict equality only when there is relative motion at contact.

\section{B. Linear Approximation of Friction Cones}

Equation 1 says that under Coulomb's law the dynamics of a mechanical system are governed, in part, by quadratic constraints. The convention is to replace the friction cone $F C$ with a pyramidal approximation [3] where each face of the approximated cone represents a linear constraint.

To do so, we replace the original basis of the tangent plane at contact $\langle\hat{\boldsymbol{t}}, \hat{\boldsymbol{o}}\rangle$ by a finer discretization $\left\langle\hat{\boldsymbol{d}}_{1}, \hat{\boldsymbol{d}}_{2} \ldots \hat{\boldsymbol{d}}_{g}\right\rangle$. We chose the discretization so that for each generator $\hat{\boldsymbol{d}}_{i}$, its antipodal $-\hat{\boldsymbol{d}}_{i}$ is also a generator. Consequently, we can always express the frictional force as a positive linear combination $\sum \beta_{j} \hat{\boldsymbol{d}}_{j}$. Note that each generator $\hat{\boldsymbol{d}}_{j}$ is the projection of an edge of the pyramidal approximation to the tangent plane, hence the total contact force can be expressed in local coordinates as $\overline{\boldsymbol{\Lambda}}=\left[\lambda_{n}, \beta_{1}, \beta_{2} \ldots \beta_{g}\right]^{\top}$.

Collect all generators $\hat{\boldsymbol{d}}_{j}$ in a matrix $\boldsymbol{D}$ and the magnitudes along those components in a vector $\boldsymbol{\beta}$, we then approximate the friction cone as:

$$
\overline{F C}=\left\{\lambda_{n} \hat{\boldsymbol{n}}+\boldsymbol{D} \cdot \boldsymbol{\beta} \mid \lambda_{n} \geq 0, \boldsymbol{\beta} \geq 0, \boldsymbol{e}^{T} \boldsymbol{\beta} \leq \mu \lambda_{n}\right\}
$$

where $e^{\top}=[1 \ldots 1]$

Coulomb's law also specifies that the friction force should be on top of the friction cone if and only if there is relative motion at contact. To impose it, we introduce a slack variable $\xi$ that intuitively represents the magnitude of the relative tangential acceleration at a contact. Then we impose:

$$
\left[\mu \lambda_{n}-\boldsymbol{e}^{\top} \boldsymbol{\beta}\right] \xi=0, \quad \mu \lambda_{n}-\boldsymbol{e}^{\top} \boldsymbol{\beta} \geq 0, \quad \xi \geq 0
$$

which guarantees that only when there is relative motion at contact $(\xi>0)$, the friction force can lie on the edge of the friction cone $\left(\mu \lambda_{n}-\boldsymbol{e}^{\top} \boldsymbol{\beta}=0\right)$.

\section{Linear Approximation of Maximum Dissipation Principle}

The maximum dissipation principle relates frictional forces and motions at each individual contact. It states that friction is always along the direction that maximizes energy dissipation, which intuitively means that friction will oppose-as much as possible - the direction of motion.

We follow Stewart and Trinkle [25] to express the principle as a linear complementarity condition:

$$
\left[\xi \boldsymbol{e}+\boldsymbol{D}^{\top} \vec{a}\right]^{\top} \boldsymbol{\beta}=0, \quad \xi \boldsymbol{e}+D^{\top} \vec{a} \geq 0, \quad \boldsymbol{\beta} \geq 0
$$

where, again, $\xi$ approximates the magnitude of the relative tangential acceleration at contact and $\vec{a}$ its relative acceleration in the positive basis $\left\langle\hat{\boldsymbol{d}}_{1}, \ldots \hat{\boldsymbol{d}}_{g}\right\rangle$. Jointly, (2), (3), and (4) impose the friction force to maximally oppose the tangential acceleration, which will usually end up lying along one of the generators if the contact slides.

\section{Contacts with non-trivial Geometry}

Simple point contact models are usually favored because of computational convenience. However, they often fall short when reproducing physical contact realistically. A line or a planar contact kinematically constrains the set of relative motions between object and finger more than a point contact does. Also, since the normal force can be distributed unevenly across the contact, the effective frictional force might depend on the direction of motion of the bodies in contact.

On the other hand, more complex contact models for surface contacts such as the limit surface by Goyal [14] can produce accurate frictional behavior, but are computationally very expensive.

In this work, we approximate a complex contact as a discrete set of rigidly-connected and evenly-distributed hard point contacts as shown in Fig. 4. Both the relative accelerations and the normal forces at the constituent contacts must be compatible with the fact that the points are rigidly attached to each other. For example, in the case of a rigid line contact, the knowledge of the acceleration at two constituent contact points is sufficient to determine the acceleration at
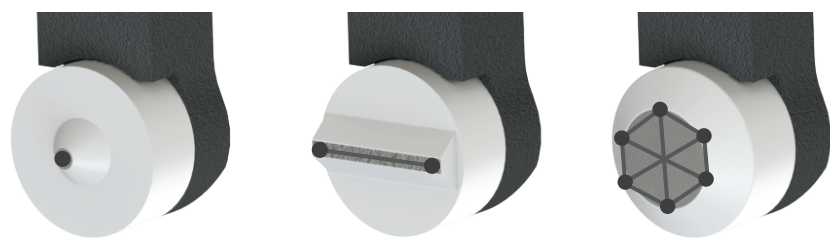

Fig. 4. Different contact geometries: point, line and planar, modeled as sets of rigidly connected $k$ point; vertex $(k=1)$, edge $(k \geq 2)$, face $(k \geq 3)$ 
any other point on the line. Similarly, in the case of a planar contact, three points determine the behavior of the entire patch. In general, for a contact of dimension $m$ (1-line, 2planar), discretized into $k$ constituent points, we impose:

- $k$ friction cone constraints and $k$ maximal dissipation constraints, one at each constituent contact, relating their respective forces and accelerations.

- $3 k-m-4$ independent constraints relating the accelerations of the $k$ constituent points, to impose they move rigidly attached to each other. For example, for the case of a line contact $(m=1)$, given the accelerations $\vec{a}_{1}$, $\vec{a}_{2}$ at two constituent points $p_{1}, p_{2}$, the acceleration $\vec{a}_{j}$ at any other constituent point $p_{j}$ must satisfy:

$$
\overrightarrow{a_{j}}=\vec{a}_{1}+\frac{\vec{a}_{2}-\overrightarrow{a_{1}}}{\operatorname{dist}\left(p_{2}, p_{1}\right)} \operatorname{dist}\left(p_{j}, p_{1}\right)
$$

yielding a total of $3(k-2)=3 k-6$ constraints. We further impose that the accelerations at points $p_{1}$ and $p_{2}$ have equal projection along the axis between them, making for a total of $3 k-5$ constraints. For a planar contact, we similarly construct the constraints by linearly interpolating the accelerations from three reference points.

- The sum of the normal forces at all constituent contacts to be equal in magnitude to its desired gripping force:

$$
\sum_{j=1 \ldots k} \lambda_{n_{j}}=\text { Gripping force }
$$

\section{GRASP MODELING}

In this section we review the use of matrix analysis to study the first order stability of a grasp by a set of point contacts [24], and detail the process to augment it to consider the effect of an external pusher. Let $p_{1} \ldots p_{n}, p_{\text {ext }_{1}} \ldots p_{\text {ext }_{m}}$ be the set of all internal and external point contacts in the grasp, including all constituent points of all contacts.

\section{A. Grasp Matrix}

The grasp matrix $\mathbf{G}$ defines the span of all possible wrenches transmitted by all contacts to the object, in the object reference frame. Following the notation in Section IV-

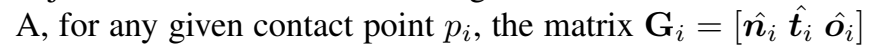
linearly spans the set of forces that contact $i$ is capable of transmitting to the object. Then the contact force transmitted by $p_{i}$ to the object is $\mathbf{G}_{i} \cdot \boldsymbol{\Lambda}_{i}$. We build grasp matrix $\mathbf{G}$ by concatenating matrices $\mathbf{G}_{i}$ 's for all the contacts:

$$
\mathbf{G}=\left[\begin{array}{llll}
\mathbf{G}_{1} \ldots \mathbf{G}_{n} & \mathbf{G}_{\mathrm{ext}_{1}} \ldots \mathbf{G}_{\mathrm{ext}_{m}}
\end{array}\right]
$$

We collect $\boldsymbol{\Lambda}_{i}$ 's for all the contacts into a big vector $\boldsymbol{\Lambda}$ which allows us to compute the total wrench on the object as $\mathbf{G} \cdot \boldsymbol{\Lambda}$.

We can write equivalent expressions for the polyhedral approximation of friction cone, where now $\overline{\mathbf{G}}=$ $\overline{\mathbf{G}}_{i}=\left[\begin{array}{ll}\hat{n}_{i} & \hat{d}_{1_{i}} \ldots \hat{d}_{g_{i}}\end{array}\right]$ spans the set of forces that contact $i$ can transmit to the object, and $\bar{\Lambda}_{i}$ is the column vector $\left[\lambda_{n_{i}}, \beta_{1_{i}} \ldots \beta_{g_{i}}\right]^{\top}$ with their corresponding magnitudes. Analogously, we build $\overline{\mathbf{G}}$ and $\bar{\Lambda}$ by concatenating them for all contacts. Then, the contact force transmitted by point $i$ is $\overline{\mathbf{G}}_{i} \cdot \overline{\mathbf{\Lambda}}_{i}$, and the total wrench applied on the object $\overline{\mathbf{G}} \cdot \overline{\boldsymbol{\Lambda}}$.
With this notation, we can finally write down the condition for the stability of the grasp (force balance on the object) as:

$$
\overline{\mathbf{G}} \cdot \overline{\mathbf{\Lambda}}+\vec{w}=\mathbf{M} \cdot \vec{a}_{\text {obj }}
$$

where $\vec{w}$ is the gravitational wrench applied on the object, $\mathbf{M}$ is the generalized inertia matrix, and $\vec{a}_{\text {obj }}$ is the acceleration of the object in the object frame.

\section{B. Hand Jacobian Matrix}

The hand jacobian matrix $\mathbf{J}$ encodes the motion of actuator joints into local motions at contact points. We construct it as $\mathbf{J}^{\top}=\left[\mathbf{J}_{1}^{\top} \ldots \mathbf{J}_{n}^{\top}\right]$, where $\mathbf{J}_{i}$ has one column for each hand actuator and expresses the induced local velocity at point contact $p_{i}$ in the local frame $\left\langle\hat{n}_{i}, \hat{t}_{i}, \hat{o}_{i}\right\rangle$. We extend the hand jacobian to include the effect of the external pusher discretized into $m$ points $p_{\text {ext }_{1}} \ldots p_{\text {ext }_{m}}$ like:

$$
\mathbf{J}^{\top}=\left[\begin{array}{llllll}
\mathbf{J}_{1}^{\top} & \ldots & \mathbf{J}_{n}^{\top} & \mathbf{J}_{\operatorname{ext}_{1}}^{\top} & \ldots & \mathbf{J}_{\text {ext }_{m}}^{\top}
\end{array}\right]
$$

where, under the assumption that the hand is moved by a robot arm with full 6DOF workspace dexterity, we model the virtual actuation of the first external contact as the identity matrix $\mathbf{J}_{\text {ext }_{1}}^{\top}=\mathbb{I}_{6}$. The jacobian matrices of all the other external points are reflections of the kinematic change from their corresponding local frames to the frame at the first contact. Note that relying on the arm kinematics gives us full dexterity over that contact point, which ultimately provides a level of dexterity and accuracy hard to achieve in traditional in-hand manipulation limited to gripper dexterity.

\section{Contact Accelerations}

The local accelerations at contacts are related to the accelerations of the object, hand, and pusher. We can look at the motion of a contact point from two perspectives:

- From the object point of view, the grasp matrix relates the acceleration of the object to the acceleration at all contacts as $\mathbf{G}^{\top} \cdot \vec{a}_{\text {obj }}$.

- From the hand point of view, the hand jacobian matrix relates the accelerations of the actuators $\ddot{\theta}$ to the accelerations at all contacts as $\mathbf{J} \cdot \ddot{\theta}$.

Note that $\ddot{\theta}$ includes both the motion of the actuators of the gripper, which (although not necessary) we will assume to be zero, and the "virtual" actuators of the pusher, for which the Jacobian is the identity. The relation formulates then as:

$$
\vec{a}=\mathbf{G}^{\top} \cdot \vec{a}_{\mathrm{obj}}-\mathbf{J} \cdot \overrightarrow{\ddot{\theta}}
$$

\section{The Mechanics of Prehensile Pushing}

In this section we summarize our model of the mechanics of prehensile pushing. We assume that the pushing operation starts from a static grasp, and that the pushing speed is slow enough that frictional forces dominate over inertiathe quasi-dynamic assumption. The quasi-dynamic nature of the formulation allows us to describe all constraints directly in terms of contact forces and contact accelerations. The following sections sums up the set of constraints that define prehensile pushing, and describes an approach to find a solution. 


\section{A. Constraints}

Prehensile pushing is subject to these constraints:

Newtonian mechanics. In response to the pusher force, all contacts develop frictional forces. Following Newton's law, the acceleration of the object must be in accordance with the object wrench resultant of finger forces, pusher forces and gravity. Equation (8) describes the force imbalance:

$$
\overline{\mathbf{G}} \cdot \overline{\mathbf{\Lambda}}+\vec{w}=\mathbf{M} \cdot \vec{a}_{\mathrm{obj}}
$$

Rigid body constraints and Motion of the pusher. The object is rigid, so all contacts must move in synchrony. Equation (10) relates the local contact accelerations $\vec{a}$ to the object, and pusher accelerations:

$$
\vec{a}=\mathbf{G}^{\top} \cdot \vec{a}_{\mathrm{obj}}-\mathbf{J} \cdot \overrightarrow{\ddot{\theta}}
$$

Unilateral contacts. Contacts can only push, not pull, and they can do so only when there in no separation between the contacting bodies at the contacts. Further, the acceleration at contact must not be such that it will produce penetration. We can express both conditions as a linear complementarity condition on the normal components of the accelerations and forces at each contact as:

$$
a_{n} \cdot \lambda_{n}=0, a_{n} \geq 0, \lambda_{n} \geq 0
$$

Frictional force. Section IV-B and Section IV-C shows how Coulomb's law and the principle of Maximal Dissipation can be both approximated by the combination of two linear complementarity constraints for each contact:

$$
\begin{gathered}
{\left[\mu \lambda_{n}-\boldsymbol{e}^{\top} \boldsymbol{\beta}\right] \xi=0, \quad \mu \lambda_{n}-\boldsymbol{e}^{\top} \boldsymbol{\beta} \geq 0, \quad \xi \geq 0} \\
{\left[\xi \boldsymbol{e}+\boldsymbol{D}^{\top} \vec{a}_{\mathrm{obj}}\right]^{\top} \boldsymbol{\beta}=0, \quad \xi \boldsymbol{e}+D^{\top} \vec{a}_{\mathrm{obj}} \geq 0, \quad \boldsymbol{\beta} \geq 0}
\end{gathered}
$$

Contacts with complex geometries. Section IV-D discusses how to approximate contacts with non-trivial geometry by discretizing them into a set of rigidly attached hard point contacts. This leads to $3 k-m-4$ linear constraints relating the accelerations of the constituents contacts of the patch. And we also impose the total desired gripping force at each contact to be equal to the sum of the forces at each constituent point:

$$
\sum_{j=1 \ldots k} \lambda_{n_{j}}=\text { Gripping force }
$$

\section{B. Optimization Problem}

The collection of all constraints define a solution space for contact forces, contact accelerations, and object acceleration. The problem has the form of a linear complementarity problem (LCP) [26] with the addition of extra linear constraints. This is commonly referred to as mixed LCP where some of the variables are subject to complementarity constraints and others are not. We solve it by reformulating it as a quadratic optimization problem. We rewrite all complementarity constraints as a quadratic cost function to minimize subject to the rest of linear equality and inequality constraints, forming
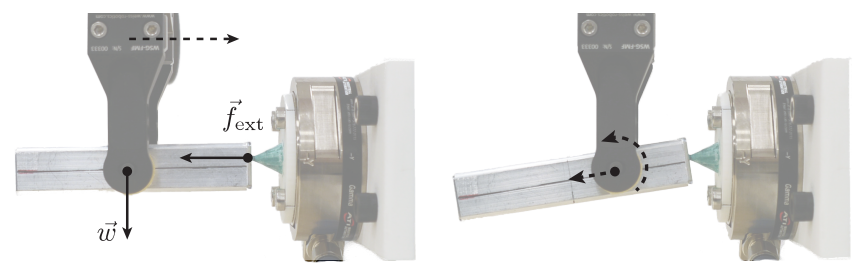

(a) Sliding with point contact.
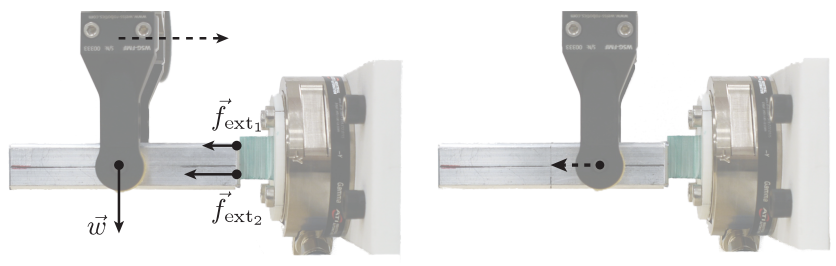

(b) Sliding with line contact.

Fig. 5. The figure shows the beginning (left) and end (right) of a straight sliding prehensile push. The dashed arrows in the figures on the right represent the twist of the object with respect to the gripper. (a) Under gravity $(\vec{w})$, an external point pusher cannot produce a straight slide. (b) The stabilizing effect of a line pusher can be explained by a couple of contact forces whose magnitudes adjust to balance the effect of gravity, and add up to the total pushing force.

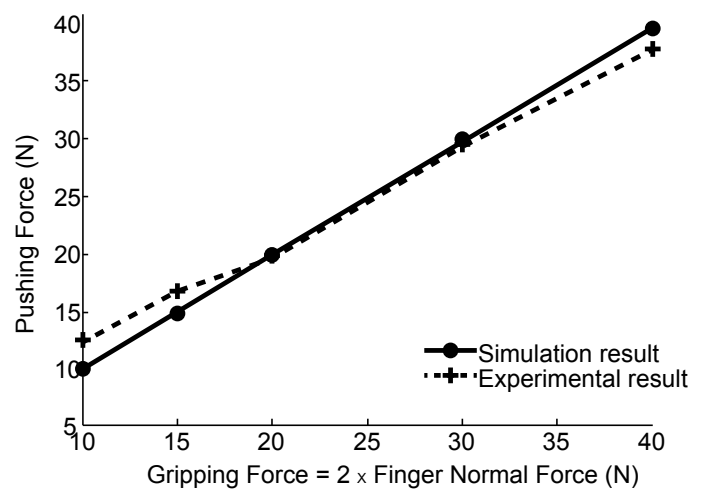

Fig. 6. Predicted and experimental pushing force required to break the grasp in the setup in Fig. 5(b) when changing the gripping force.

a convex solution space. As each complementarity term is constrained to be non-negative, the minimum occurs when complementarity constraints go to zero.

We use MATLAB's interior point solver to find a solution. The solution to the optimization directly gives the minimum force required at the pusher to move the object and the instantaneous motion of the object in the hand.

\section{Primitive Prehensile Pushing Actions}

In this section we apply the algorithm to three primitive prehensile pushing actions: sliding, pivoting and rolling. In general, these primitives can superimpose, although in this paper we study them in isolation. For each of the primitive actions, we describe results from the proposed model and compare the predictions to observations from real experiments. We instrument the real experiments with a forcecontrolled parallel-jaw gripper, and a force/torque sensor sitting behind the contact in the environment to monitor the contact forces. 

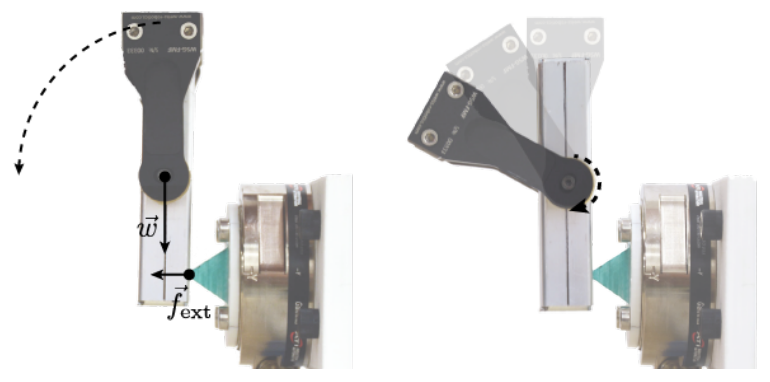

Fig. 7. The figure shows the beginning (left) and end (right) of a pivoting prehensile push. The gripper rotates about the fingertips. Constrained by the external line contact, the object stays in the same pose with reference to the world frame. From a different perspective, the pusher (the external line contact) can be seen as rotating about the fingertips while the gripper is fixed, forcing the object to rotate about the fingertips as shown in the figure on the right.

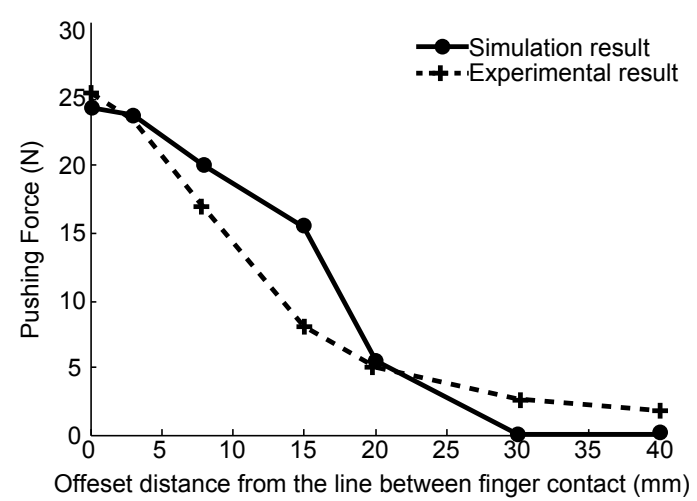

Fig. 8. Variation in the pushing force as the pusher location is changed for the setup in Fig. 7.

\section{A. Straight Sliding}

This primitive action pushes an object in a straight line, with the goal of producing an equally straight motion on the object. Fig. 5 illustrates the example of pushing a rectangular object held by a two-finger gripper.

Our formulation predicts that if the pusher makes a point contact with the object, under the effect of gravity, it is hard to push the object straight and object will tend to rotate while sliding. Straight sliding is possible only for a very specific location below the center of gravity of the object. Instead, when pushed with a line contact, the resulting motion of the object is always straight. As illustrated in Fig. 5, our model shows that, indeed, for the case of a line pusher modeled as two rigidly connected points at its ends, a pair of coupled forces both pushing straight but with different magnitudes are responsible for compensating for gravity.

These predictions from the simulation are quite intuitive and were comparable with the experimental observations, an instance of which is shown in Fig. 5. Overall, our model explains well the differences between a point and a line pusher, in particular the stabilizing effect of the geometry and the robustness against the contact location. Although not shown here, a planar contact shows a similar effect in simulation as well as in practice.

We also evaluated the effects of changing the grip force.

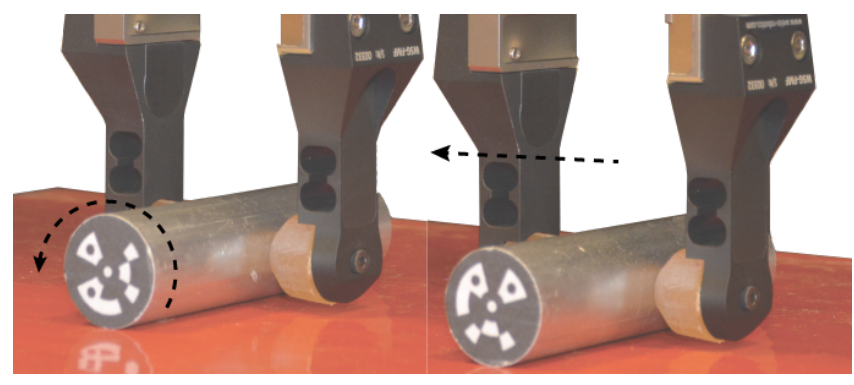

Fig. 9. The figure shows start (right) and end (left) instances of rolling primitive. As the gripper moves to the left with loosely holding a cylindrical object in the fingers (right), high friction between the tabletop surface and the object forces the object to roll in the fingers (left).

Fig. 6 shows that our model captures well the observed experimental linear relationship between the gripping force and the pushing force required to break the grasp.

\section{B. Pivoting}

Pivoting refers to the action of forcing an object to rotate about the axis between finger contacts as in Fig. 7. The action is similar to sliding, but the selection of the location and motion of the external contact favors a reorientation rather than a translation on the object.

To gain controllability over the action, we chose planar rather than point contacts at the fingertips of the gripper, which prevents gravity from freely reorienting the object. Frictional interaction is more complex, but still predictable by our model. In particular we can explain the variation in the required force to pivot the object, and its motion, with changing the contact location of the pusher.

The model correctly predicts that a line pusher rotating about the finger contacts will produce the desired pivoting. Intuitively, the further the location of the external pusher from the pivoting axis, the smaller the required force, and the more dominant the pivoting motion is over possible undesired linear slip. Fig. 8 shows the force variation as predicted by the models, as well as observed in experiments.

\section{Rolling}

The rolling primitive, starts from a grasp on a prismatic or cylindrical object and has the goal of rotating the part along its longitudinal axis.

Consider a case where a cylindrical object is placed on a sheet of some material and held loosely with two parallel fingers on the curved surface of the cylinder. If we try to pull the sheet under the object while keeping the hand still, the object can either slide or roll or undergo a combination of sliding and rolling over the sheet. We observed the same behavior with the rolling primitive.

Our formulation predicts that at low coefficient of friction between the object and the external contact, the object purely slides over the external contact with no rolling; however, as we increase the coefficient of friction, tendency of the object to roll increases, leading to pure rolling above certain coefficient of friction. The similar effect is observed if the 
gripping force is varied from high to low while keeping the coefficients of friction constant.

To reliably implement the rolling primitive we had to use a material of very high coefficient of friction on a tabletop and loose grip at the finger contacts, followed by a motion of the gripper parallel to the tabletop, as shown in Fig. 9. As expected, it was observed that if we grasp the object harder, the object tends to slide over the friction surface instead of rolling.

\section{DISCUSSION AND FUTURE WORK}

In this paper we explore in-hand manipulation of a grasped object by pushing it against its environment. We focus on a quasi-dynamic analysis of the motion of the pushed object: given a grasp configuration, gripping forces, and the location and motion of a pusher, our formulation estimates the minimum force required from the pusher to move the object in the grasp and the instantaneous motion of the object. To do so, we formulate a complementarity problem by supplementing the contact and grasp modeling techniques with computational models of the kinematic and frictional effects of patch contacts, as well as the guiding motion of the pusher.

We demonstrate the application of the proposed method with three primitive prehensile pushing actions: sliding, pivoting and rolling. We characterize these actions for different types of pusher contacts. Simulation and experimental results corroborate that the outcome of a prehensile push is affected by contact geometry and can be made more predictable and reliable by engineering the shape and location of the contact.

Future work involves extending the presented instantaneous analysis of forces and accelerations, to continuous motions and to optimize the location and motion of the pusher for a desired reorientation of the grasped object. The computational/optimization nature of the formulation for the mechanics of prehensile pushing presented here is a key enabler for it.

We are particularly interested in the possible role of the geometry of contact on funneling down the possible set of motions of the pushed object, which can play a key role in stabilizing prehensile pushes. In future work, we hope to investigate the algorithmic design of contact shapes for robot fingers and the environment around them to enable faster, simpler and more reliable in-hand manipulation. The ability to plan and design for stable prehensile pushes will contribute to enabling robots to manipulate with precision and confidence.

\section{REFERENCES}

[1] N. Chavan Dafle, A. Rodriguez, R. Paolini, B. Tang, S. Srinivasa, M. Erdmann, M. Mason, I. Lundberg, H. Staab, and T. Fuhlbrigge, "Extrinsic dexterity:in-hand manipulation with external forces," in IEEE Int Conf. on Robotics and Autom., 2014.

[2] J. C. Trinkle, J. Pang, S. Sudarsky, and G. Lo, "On dynamic multirigid-body contact problems with coulomb friction," ZAMM - Journal of Applied Math. and Mechanics, vol. 77, no. 4, pp. 267-279, 1997.
[3] A. Goldman and A. W. Tucker, "Ployhedral convex cones," Annals of Mathematics Study Study No. 38, Princeton University Press, Princeton ,New Jersey, pp. 53-97, 1956.

[4] J. K. Salisbury Jr., "Kinematic and Force Analysis of Articulated Hands," PhD Dissertation, Stanford University, 1982.

[5] J. Salisbury and J. Craig, "Articulated hands: Force control and kinematic issues," Int J Robot Res, vol. 1, no. 1, pp. 4-17, 1982.

[6] R. Fearing, "Simplified grasping and manipulation with dextrous robot hands," IEEE Journal of Robotics and Autom., vol. 2, no. 4, pp. 188$195,1986$.

[7] T. Omata and K. Nagata, "Planning reorientation of an object with a multifingered hand," in IEEE Int Conf. on Robotics and Autom., 1994, pp. 3104-3110.

[8] L. Han and J. Trinkle, "Dextrous manipulation by rolling and finger gaiting," in IEEE Int Conf. on Robotics and Autom., 1998, pp. 730735 .

[9] D. Rus, "In-Hand Dexterous Manipulation of Piecewise-Smooth 3-D Objects," Int J Robot Res, vol. 18, no. 4, pp. 355-381, 1999.

[10] M. Cherif and K. Gupta, "Planning quasi-static fingertip manipulations for reconfiguring objects," in IEEE T Robotic Autom, vol. 15, 1999, pp. 837-848.

[11] D. Brock, "Enhancing the dexterity of a robot hand using controlled slip," in IEEE Int Conf. on Robotics and Autom., vol. 1, 1988, pp. 249-251.

[12] R. D. Howe and M. R. Cutkosky, "Practical force-motion models for sliding manipulation," Int Journal of Robotics Res., vol. 15, no. 6, pp. 557-572, 1996.

[13] R. Howe, I. Kao, and M. Cutkosky, "The sliding of robot fingers under combined torsion and shear loading," in Robotics and Autom., 1988. Proceedings., 1988 IEEE Intl Conf. on, Apr 1988, pp. 103-105 vol.1.

[14] S. Goyal, "Planar sliding of a rigid body with dry friction: Limit surfaces and dynamics of motion," PhD Dissertation, Department of Mechanical Engineering, Cornell University, 1989.

[15] P. R. Sinha, "A Contact Stress Model for Determining Forces in an Equilibrium Grasp," University of Pennsylvania Dept. of Computer and Information Science Tech Report No. MS-CIS-90-19, 1989.

[16] J. Trinkle and R. Paul, "Planning for dexterous manipulation with sliding contacts," Int J of Robot Res., vol. 9, no. 3, pp. 24-48, 1990.

[17] A. Cole, P. Hsu, and S. Sastry, "Dynamic control of sliding by robot hands for regrasping," Robotics and Autom., IEEE Transactions on, vol. 8, no. 1, pp. 42-52, Feb 1992.

[18] I. Kao and M. R. Cutkosky, "Quasistatic manipulation with compliance and sliding," Int J of Robot Res., vol. 11, no. 1, pp. 20-40, 1992.

[19] A. Bicchi and R. Sorrentino, "Dexterous manipulation through rolling," in IEEE Int Conf. on Robotics and Autom., 1995, pp. 452457.

[20] L. S. Hong and M. R. Cutkosky, "Fixture planning with friction," Journal of Manufacturing Science and Engineering, vol. 113(3), pp. 320-327, 1991.

[21] M. Peshkin and A. Sanderson, "Planning Robotic Manipulation Strategies for Workpieces that Slide," IEEE Journal on Robotics and Autom., vol. 4, no. 5, pp. 524-531, 1988.

[22] K. Lynch, "The mechanics of fine manipulation by pushing," in Robotics and Autom., 1992. Proceedings., 1992 IEEE Int Conf. on, May 1992, pp. 2269-2276 vol.3.

[23] K. M. Lynch and M. T. Mason, "Stable pushing: Mechanics, controllability, and planning," Int J Robot Res, vol. 15, no. 6, pp. 533-556, 1996.

[24] D. Prattichizzo and J. Trinkle, Springer Handbook of Robotics- Grasping, B. Siciliano and O. Khatib, Eds. Springer, 2008.

[25] D. E. Stewart and J. C. Trinkle, "An implicit time-stepping scheme for rigid body dynamics with inelastic collisions and coulomb friction," Intl Journal for Numerical Methods in Engineering, vol. 39, no. 15, pp. 2673-2691, 1996.

[26] R. W. Cottle and G. B. Dantzig, "A generalization of the linear complementarity problem," Journal of Combinatorial Theory, vol. 8, no. 1 , pp. $79-90,1970$. 